\title{
Revisión del cálculo de poder calorífico y punto de rocío del gas natural, y de la estimación de sus incertidumbres
}

\section{Review of calculation of calorific value and dew point of natural gas, and estimating uncertainties}

\section{Revisão do cálculo do valor calorífico e ponto de orvalho do gás natural e a estimativa de suas incertezas}

\author{
Elizabeth Ayala Blanco*; Adriana Alexandra Aparicio Ariza; Carlos Eduardo García Sánchez. \\ Grupo de Investigación en Fluidos y Energía GIFE. \\ Corporación Centro de Desarrollo Tecnológico de Gas, Parque tecnológico UIS Guatiguará Km 2 vía El Refugio. \\ Piedecuesta, Santander, Colombia. \\ “eayala@cdtdegas.com
}

Fecha Recepción: 23 de junio de 2015 Fecha Aceptación: 11 de noviembre de 2016

\begin{abstract}
Resumen
El gas natural debe cumplir ciertos requisitos que garanticen que su producción, transporte, distribución y consumo se realiza de forma segura. Estos requisitos corresponden a los parámetros de calidad de gas dentro de los cuales se encuentran el poder calorífico y el punto de rocío de hidrocarburo. Su correcta medición es de vital importancia, especialmente en los puntos de transferencia de custodia, ya que tanto quien entrega como quien recibe está interesado en conocer la calidad del gas comercializado con una alta exactitud. La presente revisión recopila las diferentes formas de medición de los parámetros de calidad de gas seleccionados, a partir de trabajos encontrados en la literatura tanto en la determinación de su valor como de su incertidumbre asociada. Sin embargo, a pesar de que los trabajos relacionados con la estimación de incertidumbre del poder calorífico y el punto de rocío de hidrocarburos son limitados, esta revisión representa un avance en la ruta de investigación aplicada en la adecuada determinación de estos parámetros y en la obtención de resultados con mayor confiabilidad metrológica de las magnitudes químicas del gas natural.
\end{abstract}

Palabras clave: gas natural, punto de rocío de hidrocarburo, poder calorífico, incertidumbre.

\begin{abstract}
Natural gas should meet certain requirements that ensure that their production, transport, distribution and consumption is carried out safely. These requirements correspond to the parameters of gas quality inside which are the calorific value and the hydrocarbon dew point. Correct measurement is of vital importance, especially in the custody transfer points, since who delivered as who receives both are interested in knowing the quality of the gas marketed with a high accuracy. This review gathers different forms of measurement of the gas quality parameters selected, from studies found in the literature both in the determination of its value and its associated uncertainty. However, while the works related to the estimation of uncertainty of the calorific value and the hydrocarbon dew point are limited, this review represents a breakthrough in the path of research applied in the proper determination of these parameters and results with greater metrology reliability of chemical quantities of natural gas.
\end{abstract}

Keywords: natural gas, hydrocarbon dew point, heating value, uncertainty.

Cita: Ayala Blanco E, Aparicio Ariza AA, García Sánchez CE. Revisión del cálculo de poder calorífico y punto de rocío del gas natural, y de la estimación de sus incertidumbres. rev.ion. 2016;29(2):87-99. 


\section{Resumo}

Gás natural deve atender a certos requisitos que garantem que sua produção, transporte, distribuição e consumo é realizado de forma segura. Essas exigências correspondem aos parâmetros do gás de qualidade no interior dos quais são o poder calorífico e o ponto de orvalho de hidrocarbonetos. Correta de medição é de vital importância, especialmente em pontos de transferência de custódia, desde que entregue como quem recebe ambos estão interessados em saber a qualidade do gás comercializado com uma alta precisão. Esta revisão reúne diferentes formas de medição dos parâmetros de qualidade de gás selecionado, de estudos encontrados na literatura tanto na determinação do seu valor e a incerteza associada. No entanto, enquanto as obras relacionadas com a estimativa de incerteza do poder calorífico e o ponto de orvalho de hidrocarbonetos são limitadas, esta revisão representa um avanço no caminho da investigação aplicada na determinação correta desses parâmetros e resultados com maior metrologia de confiabilidade das quantidades químicas do gás natural.

Palabras-chave: gás natural, hidrocarboneto ponto de orvalho, calorifico, incerteza.

\section{Introducción}

El Gas Natural (GN) es una compleja mezcla de sustancias principalmente conformada por hidrocarburos ligeros, que adicionalmente puede incluir hidrocarburos pesados, agua, sulfuro de hidrógeno y dióxido de carbono, entre otros componentes. Constituye uno de los combustibles más usados actualmente, y su importancia en la canasta energética mundial ha ido en aumento como sustituyente de combustibles como el carbón, queroseno y el petróleo, respecto a los cuales es una fuente de energía menos agresiva para el medio ambiente, al generar menos material particulado y menor cantidad de dióxido de carbono por unidad de energía obtenida [1]. Estimaciones del 2013 mostraban que las reservas mundiales del GN se encontraban distribuidas de la siguiente manera: Oriente Medio 43,2\%, Europa y Eurasia $30,5 \%$, Asia y el pacífico 8,2\%, África 7,6\%, Norte América $6,3 \%$, y Sudamérica y Centroamérica cada una $4,1 \%$ [2].

La comercialización del GN depende de su Poder Calorífico (PC), que corresponde al principal indicador para establecer su precio y del cumplimiento de restricciones sobre el valor de ciertos parámetros que tienen impacto sobre la seguridad de los procesos de transporte y uso del GN, como el Punto de Rocío de Hidrocarburo (PRHC) [3]. Tales parámetros son función de la composición del GN, y en ocasiones la legislación en diferentes zonas geográficas establece límites permisibles distintos para cada uno de ellos, razón por la cual, ha sido necesaria la creación de normas internacionales para contribuir a eliminar las barreras técnicas en el comercio y transporte del GN [4-7].
EI PC se define como la cantidad de calor liberado por la combustión completa a condiciones estándar establecidas por cada país de una unidad de volumen del gas a condiciones de referencia, normal en el caso de Europa $(273,15 \mathrm{~K}$ y 1013,25mbar) y estándar para el caso de Estados Unidos (288,15K y 1013,25mbar)[8]. Existen dos maneras de presentar esta propiedad: Poder Calorífico Superior (PCS), que es el PC que se calcula considerando que el agua resultante de la combustión se encuentra en estado líquido (condensado), y Poder Calorífico Inferior (PCI), que es el PC resultante al asumir que el agua producto de la combustión está en estado vapor [9]. Su determinación se puede realizar de manera directa midiendo el PCS usando calorímetros o equipos de combustión catalítica, o de manera indirecta basándose en la identificación de la composición obtenida por análisis cromatográfico [10], y se pueden presentar diferencias en orden de magnitud al ser expresado el PC a condiciones normales $\left(\mathrm{MJ} / \mathrm{m}^{3}\right)$ o estándar $\left(\mathrm{kcal} / \mathrm{m}^{3}\right)$.

EI PRHC también depende de la composición del GN y se define como la temperatura por encima de la cual no se presenta condensación detectable de hidrocarburos a una presión específica [11]. En la industria del GN su control tiene como objetivo prevenir la formación de condensados, los cuales son nocivos para los equipos e instalaciones del gas al ocasionar corrosión, combustión incompleta, obstrucciones y daños por impacto. Por lo tanto, en la cadena del GN es necesario determinar adecuadamente el PRHC y ajustarlo si es necesario, para poder garantizar la seguridad y fiabilidad de los sistemas de transporte, distribución y consumo del GN. La determinación del PRHC puede hacerse de dos maneras: 
directamente, por medio de la detección de la formación de una película de condensado sobre una superficie que se enfría de manera controlada y que se pone en contacto con el gas natural a la presión de interés, e indirectamente, cuantificando la composición del GN por medio de un cromatógrafo de gases en línea o en el laboratorio y posteriormente estimando el punto de rocío usando cálculos de equilibrio de fases [12].

Junto al resultado de cualquier medición, incluyendo el cálculo de cualquier característica física o química de un fluido, se debe reportar la incertidumbre de la medición, que consiste en la duda que se tiene sobre el valor de una medida en particular. La incertidumbre cuantifica la confianza que se tiene en un resultado de medición, y su estimación es fundamental en la toma de decisiones y la comparación con respecto a otros resultados de la misma índole que ejecutan laboratorios y entes gubernamentales, industriales y comerciales [13]. Particularmente para el caso del GN, la estimación de la incertidumbre de medición reduce las discrepancias entre productor y transportador en los puntos de transferencia de custodia, brindando mayor soporte a los resultados obtenidos, sobre todo si se tiene en cuenta que las propiedades termodinámicas del combustible definen su valor comercial [14].

\section{Determinación de PC y PRHC}

\section{Medición Directa}

Poder calorífico. La medición del PC del GN se puede llevar a cabo por métodos directos destructivos, en los que se realiza la combustión del GN para su determinación. Ulbig et al. [10] clasifican dichos métodos en tres grupos, de acuerdo al instrumento empleado para la medición: bomba calorimétrica, calorímetro de gases u oxidación en lecho catalítico. La combustión isocórica dentro de una bomba calorimétrica consiste en la transferencia del calor liberado por la combustión a un líquido conocido en el cual está inmersa la cámara de combustión; este método es aplicable tanto a combustibles gaseosos como a líquidos, aunque para gases tiene la desventaja de que la combustión no queda localizada en una región particular de la cámara [15-18]. El principio del calorímetro de gases es una combustión isobárica en un quemador de llama abierta, usando la diferencia de temperatura entre los gases de combustión y el aire que entra al equipo junto con los flujos de combustible y de aire para realizar el cálculo del PC [19].

En el caso de la combustión isobárica sin llama en un catalizador, se lleva a cabo la oxidación del combustible sobre un lecho catalizador sin que esta combustión genere llama, sirviendo el cambio de temperatura del lecho junto con sus dimensiones y propiedades para calcular el PC del gas [20].

También se han desarrollado equipos de flujo calorimétrico para la medición del PC, utilizando elementos Peltier para generar el calor de la llama y/o el calentador [21]. El valor calorífico se determina a partir de las mediciones de las potencias eléctricas a condiciones de flujo constante. Por otro lado, se ha propuesto un método óptico para evaluar el PC, a partir de la radiación de las llamas generadas de diferentes mezclas de gases combustibles obtenidas por un espectrómetro [22]. Se reportó que los espectros de llama del gas natural varían de acuerdo con la relación aire/combustible.

Los calorímetros isotérmicos se basan en un principio de compensación, en donde la parte principal del calorímetro es un tubo de calor. La potencia suministrada por el calentador de compensación es controlada por un sistema PID para mantener la temperatura en el interior del tubo de calor a una temperatura constante en estado estacionario. El gas es quemado en el interior del calorímetro y la entalpía de combustión se calcula a partir de la siguiente expresión:

$$
\Delta H_{\text {combustión }}=\frac{P_{\text {Base }}-P_{G a s}}{\dot{m}_{G a s}}
$$

La medición directa del poder calorífico resulta ventajosa en términos de los costos, no solo del equipo, sino también por los insumos adicionales que requiere la medición indirecta. Adicionalmente, los calorímetros no son sensibles a la composición del gas combustible, dado su principio de medición.

Punto de rocío de hidrocarburo. El PRHC se puede medir a través de equipos de espejo enfriado, ya sean manuales o automáticos. En estos equipos una muestra del gas a la presión de interés se enfría lentamente hasta que se observa aparición de condensado, registrándose la temperatura a la que esto ocurre lo cual se conoce como el Punto de Rocío de Hidrocarburo. En el equipo manual, la detección del condensado es efectuada por un operador, y por consiguiente se requiere que éste posea la habilidad, un buen 
entrenamiento y criterio para que la medición sea adecuada [23-24]. En el caso del equipo automático, la determinación del PRHC se efectúa sin intervención directa de humanos, detectándose la formación de condensado por medio de la medición de propiedades ópticas del espejo [25]. Algunos estudios, como el reporte AS 3 del National Physical Laboratory del Reino Unido, han comparado el desempeño de los equipos manuales y los automáticos. En este trabajo los autores detectaron que el equipo automático probado arroja valores más altos de PRHC respecto al equipo manual al ser utilizados con gases naturales reales, con diferencias entre 3,2 y $7,7 \mathrm{~K}$ en las pruebas efectuadas [26].

\section{Medición Indirecta}

Composición. Las determinaciones indirectas tanto del PC como del PRHC requieren la medición de la composición del GN. Esta determinación de la composición se puede realizar usando diferentes técnicas analíticas, entre las cuales la más usada es la Cromatografía de Gases (CG), que puede estar acoplada a espectrometría de masas.

La cromatografía de gases es una técnica de separación física en donde los componentes de la muestra se distribuyen entre dos fases, una estacionaria y una móvil. La muestra se inyecta al equipo, y la fase móvil transporta la muestra volatilizada a través de la fase estacionaria que generalmente es la columna cromatográfica [27]; esto permite separar los compuestos y emplear los tiempos y volúmenes de retención para la identificación cualitativa y cuantitativa. Los dispositivos comúnmente usados para identificar los compuestos del GN son el Detector de Ionización de Llama (FID) el cual es selectivo para los hidrocarburos, y el Detector de Conductividad térmica (TCD) el cual no es selectivo y permite detectar cualquier componente diferente al gas portador, por lo que es conocido como detector universal. Para determinar la composición de cada sustancia en el GN es necesario realizar una calibración del equipo efectuando ensayos cromatográficos a Materiales de Referencia (MR), los cuales son materiales en donde uno o más valores de sus propiedades son homogéneos y bien definidos (en este caso, el valor de la composición) [28] para utilizarlos en la evaluación de la relación entre el valor de la propiedad y la respuesta del equipo de medición.

La determinación de la composición por medio de la cromatografía de gases acoplada a un espectrómetro de masas usa el movimiento de los iones en campos eléctricos y magnéticos, que permite clasificar los componentes de acuerdo a su relación masa/carga. Así se obtiene información cualitativa y cuantitativa acerca de la composición atómica y molecular de materiales orgánicos e inorgánicos, confirmando los resultados obtenidos por el CG y evidenciando si hay o no presencia de impurezas [29].

Poder Calorífico. El método indirecto de determinación del PC se basa en el hecho de que cada componente del GN aporta al PC total de acuerdo a su naturaleza y su cantidad dentro de la mezcla [9]. EI PC bruto o ideal es una característica propia de un gas ideal bajo la suposición de que el agua se condense en su totalidad [30]. De acuerdo a lo anterior, el PC ideal se calcula con el fin de determinar la cantidad hipotética de energía que el gas debería entregar al ser quemado y se obtiene sumando la contribución de cada sustancia de la mezcla al PC, de modo que se realiza la multiplicación del poder calorífico de cada constituyente puro por su respectiva concentración dentro del GN y se suman los resultados, como se observa en la Ecuación 2 [30-31].

$$
P C_{i d}=\sum_{i=1}^{n} X_{i} * P C_{i}
$$

Donde $P C_{i d}$ corresponde al poder calorífico ideal de la mezcla gaseosa en términos volumétricos (energía sobre volumen), $P C_{i}$ es el poder calorífico ideal de cada componente $i$ presente en el GN, y $X_{i}$ es la fracción molar del componente $i$ presente en el GN. Sin embargo, en la realidad los gases no se comportan de acuerdo a las características de un gas ideal, razón por la cual el PC volumétrico debe corregirse teniendo en cuenta el factor de compresibilidad [31-34]; en la Ecuación $3 P C_{r}$ es el poder calorífico real del GN y $Z_{\text {mix }}$ corresponde al factor de compresibilidad de la mezcla gaseosa.

$$
P C_{r}=\frac{P C_{i d}}{Z_{m i x}}
$$

El factor de compresibilidad es un parámetro de ajuste creado para representar sustancias en fase gaseosa con una ecuación similar al modelo de los gases ideales, y se define como se muestra en la Ecuación 4 [33]. 


$$
Z_{\text {mix }}=\frac{P V}{R T}
$$

La mayoría de las correlaciones para el cálculo de $Z$ parten de la ley de los estados correspondientes, es decir, $Z$ se expresa como una función de la temperatura reducida y la presión reducida [35]. La primera herramienta de amplia aplicación en la industria del GN fue la carta desarrollada por Standing y Katz, a pesar de que su utilización llevaba asociada una alta incertidumbre [36] y representaba un trabajo tedioso y costoso [35].

Poco a poco empezaron a aparecer los métodos computacionales, mucho más prácticos en contraste con la carta. Fayazi et al. [37] y Kumar [38] resumen brevemente algunos métodos comunes para el cálculo del factor de compresibilidad, como las ecuaciones de estado entre las cuales destacan la de Peng-Robinson (PR) y la de Soave-RedlichKwong (SRK) [28,30,38], y algunas correlaciones empíricas como la de Beggs y Brill [30], el enfoque empleado por la Shell Oil Company [38], y las correlaciones de Heidaryan et al. [40], de Azizi et al. [34], y de Sanjari y Lay [41].

Sin embargo, cabe resaltar que, en la industria del $\mathrm{GN}$, el $Z$ se puede determinar bien sea siguiendo los lineamientos expresados en el reporte AGA 8 [32], el cual comprende dos métodos: "método de caracterización detallado" y "método de caracterización "grueso", o de acuerdo al método GERG 2008 [42] adoptada en la norma ISO 207652/3 y tomada como estándar en Europa debido a que es considerada una norma muy exacta para el cálculo de las propiedades termodinámicas del GN a temperaturas de 270 y $330 \mathrm{~K}$ a presiones de hasta $12 \mathrm{MPa}$.

Punto de Rocío de Hidrocarburo. Los métodos más utilizados para determinar indirectamente el PRHC usan la composición y las ecuaciones de estado para modelar el comportamiento termodinámico del GN y determinarlo a través del cálculo de equilibrio de fases. Diferentes estudios han presentado comparaciones entre valores de PRHC obtenidos con diversas ecuaciones y mediciones experimentales, para evaluar sus rangos de validez y la exactitud de los resultados obtenidos. Nasrifar et al. evaluaron 15 ecuaciones de estado en gases sintéticos con bajo contenido de hidrocarburos de orden superior, y muestras de gases naturales con alto contenido. Los resultados de PRHC obtenidos mostraron que la familia de las ecuaciones de estado Redlich-Kwong
(RK) predicen mejor a los gases sintéticos y las ecuaciones de estado de la familia Patel-Teja (PT) las muestras de GN [43]. Posterior a ello el mismo grupo realizó un estudio del modelado termodinámico de mezclas de GN por medio de una nueva ecuación de estado cúbica de dos constantes, resultante de modificar la función a (parámetro de atracción dependiente de la temperatura) en la ecuación SRK, involucrando nuevos parámetros b1, b2 y b3 y determinando el parámetro que depende del factor acéntrico mediante la correlación de la presión de vapor de las sustancias puras entre el punto triple y la temperatura en el punto crítico. La ecuación de estado empleada demostró superioridad en comparación con otras ecuaciones evaluadas y los PRHC obtenidos fueron similares a los de la familia RK [44]. Varios modelos empíricos se han propuesto para modelar el PRHC en diversas situaciones. Por ejemplo, un modelo para estimar la presión de rocío para yacimientos de gas condensado como una función de medición rutinaria de análisis de gas y temperatura de yacimiento se desarrolló a partir de 340 muestras de gas condensado [45]. El desempeño de la correlación para modelar el PRHC fue comparado con valores obtenidos de ecuaciones de estado (SRK y PR) y otras correlaciones, evidenciando que la predicción con este modelo depende de la ecuación de estado, la caracterización de fracciones pesadas y el número de pseudo-componentes. Por lo tanto con el modelo planteado se minimizó el error en el cálculo del PRHC. Otra forma particular de determinar el PRHC es utilizando la ecuación de estado GERG 2008 [42], la cual está basada en una aproximación explícita de multifluidos que involucra la energía de Helmholtz reducida. Galatro y Marín-Cordero [46] compararon los resultados obtenidos al aplicar diferentes ecuaciones de estado, y la influencia que pueden llegar a tener otros parámetros en la determinación del PRHC e incluso, un estudio reciente realizado por Jarrahiana y Heidaryan [47] propusieron una ecuación de estado que no está ligada directamente a la composición del GN, lo cual representa una ventaja para realizar aproximaciones rápidas de esta propiedad.

\section{Estimación de incertidumbre}

Debido a diversas fuentes de variabilidad que se presentan en las mediciones, se hace imposible conocer con una certeza absoluta el valor verdadero de una magnitud. Por lo tanto, resulta fundamental estimar el valor de la incertidumbre 
asociada a una medición particular para evaluar la confiabilidad de los resultados, y para esto es necesario definir correctamente el mensurando y evaluar los factores que influyen en él [48-49].

La estimación de incertidumbre puede realizarse usando diferentes enfoques matemáticos [50], de los cuales los más importantes son la metodología descrita en la Guía para la Expresión de la Incertidumbre de Medición (GUM) [51], y el método Monte Carlo (MC) [52]. Independientemente del enfoque usado, las etapas del proceso de estimación de incertidumbre son (a) formulación, que incluye la determinación del modelo de medición que relaciona al mensurando con las magnitudes de entrada y la asignación de distribuciones de probabilidad a dichas magnitudes, (b) propagación, que consiste en calcular cómo se propaga la incertidumbre a través del modelo de medición, y (c) resumen, que consiste en obtener el cálculo de la incertidumbre del mensurando a partir de los resultados de la etapa anterior; los dos enfoques de estimación mencionados difieren en la etapa de propagación [53]. La incertidumbre del mensurando puede declararse como incertidumbre combinada, que es la desviación estándar de la distribución de probabilidad calculada para el mensurando, o como incertidumbre expandida, que consiste en la mitad de la longitud de un intervalo de cobertura que se espera contenga a un porcentaje determinado de los valores que podrían atribuirse razonablemente al mensurando [51]. La formulación comienza con la definición del mensurando, que es la variable de interés en la medición, y la identificación de las magnitudes de entrada, que son las variables que afectan al mensurando. Luego se determina el modelo de medición, que consiste en un conjunto de expresiones matemáticas que relaciona a las magnitudes de entrada $X_{1}, X_{2} \ldots X_{N}$ con el mensurando $Y$, como lo muestra la Ecuación 5 [51].

$$
Y=f\left(X_{1}, X_{2}, \ldots X_{N}\right)
$$

La etapa de formulación finaliza con la representación de las magnitudes de entrada por medio de distribuciones de probabilidad, las cuales se asignan con base en la información disponible, que puede ser experimental o de otra índole.

La propagación de la incertidumbre y el posterior resumen en el método GUM se llevan a cabo aproximando el modelo de medición por medio de series de Taylor, habitualmente truncando la serie en los términos de primera derivada y despreciando la correlación entre magnitudes de entrada. A partir de esta simplificación, la incertidumbre combinada se calcula como se muestra en la Ecuación 6. Si se considera la existencia de correlaciones entre las magnitudes de entrada, el cálculo de la incertidumbre combinada se representa según la Ecuación 7.

$$
u_{c}^{2}(y)=\sum_{i=1}^{n}\left[\frac{\partial f(X)^{2}}{\partial X_{i}}\right] * u^{2}\left(X_{i}\right)
$$

$$
u_{c}^{2}(y)=\sum_{i=1}^{n}\left[\partial f\left(X^{2}\right) / \partial X_{i}\right] * u^{2}\left(X_{i}\right)+\sum_{i=1}^{n} \sum_{j=1}^{n} \frac{\partial f(X)}{\partial X_{i}} * \frac{\partial f(X)}{\partial X_{j}}
$$

Donde $u_{c}$ es la incertidumbre combinada y $u(X)$ es la incertidumbre de la composición del componente $i$. Por otra parte, en el método MC la propagación de la incertidumbre se lleva a cabo simulando numerosos ensayos de evaluación del mensurando, en cada uno de los cuales se toma una muestra de cada cantidad de entrada según la distribución de probabilidad que se les ha asignado y se calcula el mensurando según el modelo de medición. Los resultados de esos ensayos se utilizan para aproximar la distribución de probabilidad del mensurando, finalizando la etapa de resumen por medio del cálculo de la incertidumbre combinada como la desviación estándar de los resultados de los ensayos, o de la incertidumbre expandida basándose en la determinación de intervalos de cobertura para el mensurando [53].

\section{Incertidumbre en medición directa de PC y PRHC}

Existen relativamente pocos estudios relacionados con la incertidumbre asociada a la medición directa del PC. Enright y Fleischmann [54] estimaron la incertidumbre de un calorímetro de cono aplicando la metodología GUM a la medición de la tasa de 
calor liberado, según la norma ISO5660-1. Zhao y Dembsey [55] trabajaron en la estimación de incertidumbre en calorímetros, con miras a generar un procedimiento estándar para dicha estimación. Este último trabajo indicó que en los calorímetros de cono, entre más alta es la tasa de liberación de calor, menos es su incertidumbre relativa; sus resultados fueron validados usando simulación MC. Alexandrov [56] también utilizó la metodología GUM para la estimación de la incertidumbre en la medición del calor de combustión y de la energía térmica del metano en un calorímetro, encontrando una incertidumbre estándar relativa menor a $0,02 \%$ y una incertidumbre estándar combinada inferior a $0,04 \%$, concluyendo que su equipo puede ser considerado como un prototipo estándar de calorímetro de gas. Haloua et al. [56] validaron los resultados obtenidos por dos tipos de calorímetros: calorímetros de referencia primaria y calorímetros de campo, con los resultados obtenidos usando medición indirecta a partir de cromatografía de gases para seis mezclas de biogás sintético. Adicionalmente, estimaron la incertidumbre asociada a este método de medición del poder calorífico, estimando incertidumbres entre $0,07 \%$ y $0,49 \%$ para los calorímetros de referencia, y entre 0,18 y $2,48 \%$ para los calorímetros de campo. Los autores llegaron a la conclusión de que, estos resultados, comparados con la desviación estándar de aproximadamente $1 \%$ que se obtiene en la medición indirecta del PC por cromatografía de gases, demuestra que el método calorimétrico es apropiado para la medición del contenido energético de los combustibles renovables como el biogás. Yackow et al. [58] analizaron los equipos de espejo enfriado automático y manual para realizar una comparación y definir una relación entre las técnicas experimentales y de cálculo para determinar el PRHC. En el estudio se utilizaron tanto mezclas naturales como sintéticas, y los valores medidos tanto por el equipo manual como por el equipo automático fueron comparados con envolventes de fases obtenidas usando paquetes de software comercial. Los resultados obtenidos mostraron que los componentes aromáticos y cíclicos tienen una influencia en el valor final del PRHC y que los métodos de medición directa manual o automático eran comparables presentando diferencias entre un rango de 2 a $4 \mathrm{~K}$. Además, los autores hacen énfasis en que la incertidumbre asociada al cálculo del PRHC proviene directamente de la determinación de la composición del GN, y agrupan las incertidumbres de acuerdo a los rangos de composición. Los componentes que presentan valores más altos de incertidumbre son aquellos que se encuentran en concentraciones más bajas $(<1 \mathrm{ppm})$.

\section{Incertidumbre en medición indirecta de PC y PRHC}

Las incertidumbres en la determinación indirecta de PC y PRHC del GN dependen tanto de la incertidumbre en el cálculo de la composición del GN, como de la incertidumbre debida al modelo usado para calcular cada parámetro.

En la Guía para la Cuantificación de la Incertidumbre en las Mediciones Analíticas de EURACHEM/ CITAC (Guía EURACHEM/CITAC) [59] se afirma que las principales fuentes de incertidumbre en la medición de composición por cromatografía son la preparación de la muestra, los materiales de referencia, la calibración del cromatógrafo, y el análisis y procesamiento de datos. Pocos trabajos, como el realizado por Puglisi et al. [60], se han enfocado en la estimación de la incertidumbre asociada a la determinación de la composición por cromatografía, aunque de forma general la Guía EURACHEM/CITAC presenta una adaptación del método GUM a distintos métodos analíticos, y en la literatura se presentan algunas consideraciones para la estimación de la incertidumbre [48].

Cruz de Oliveira [61] estimó la incertidumbre de la composición de acuerdo al número de puntos usados en la calibración del cromatógrafo. Cuando la calibración se lleva a cabo en un solo punto la estimación de incertidumbre de la fracción molar $(x i)$ sin normalizar se calcula a partir de la Ecuación 8.

$$
\left(\frac{u\left(X_{i}\right)}{\left(X_{i}\right)}\right)^{2}=\left(\frac{u\left(X_{p}\right)}{\left(X_{p}\right)}\right)^{2}+\left(\frac{u\left(Y_{p}\right)}{\left(Y_{p}\right)}\right)^{2}+\left(\frac{u\left(Y_{0}\right)}{\left(Y_{0}\right)}\right)^{2}
$$

Donde $u(X)$ es la incertidumbre de la fracción no normalizada del componente $i, u\left(X_{p}\right)$ es la incertidumbre de la fracción molar del componente $i$ en el MR, $u\left(Y_{p}\right)$ es la incertidumbre del área del pico del componente $i$ en el MR y $u\left(Y_{\theta}\right)$ es la incertidumbre del área del pico del componente $i$ en la muestra analizada.

Cabe resaltar que se pudo identificar que este método solo es válido cuando los componentes no superan ciertos límites, como en el caso de componentes con fracciones molares entre 0,001 y $0,1 \%$ su porcentaje de desviación entre la fracción molar del componente de la mezcla y el MR debe ser del $\pm 100 \%$. Por otro lado, aplicaron un segundo 
método en el que utilizaron una curva analítica de multipuntos para una regresión no ponderada con características homocedásticas (prueba de Cochran), en donde se eliminan los valores atípicos obtenidos en el análisis cromatográfico usando la prueba de Grubbs. A partir de $n$ datos de una curva de calibración lineal y siendo $p$ el número de puntos de calibración medidos para determinar $Y_{0}$, la incertidumbre combinada de la concentración de la muestra fue calculada como se muestra en la Ecuación 9.

$$
u(P C)=\frac{s}{b_{1}} * \sqrt{\frac{1}{p}+\frac{1}{n}+\frac{\left(X_{0}-\bar{X}\right)^{2}}{\sum_{j=1}^{n}\left(X_{j}-\bar{X}\right)}}
$$

En donde $b_{1}$ es la pendiente $n$ el número de repeticiones, $X_{0}$ la concentración de cada componente en la muestra, $X$ el valor promedio de los diferentes estándar de calibración, y $X_{j}$ la concentración del MR. Además, este método se evaluó para verificar la linealidad mediante un análisis de regresión de residuos y por análisis de varianza.

Tsochatzidis et al. [62] estimaron la incertidumbre del PC según se muestra en la Ecuación 10.

$$
u(P C)=\sqrt{u_{p}^{2}+u_{c a l}^{2}}
$$

En este modelo, basado en el método GUM, se consideran como magnitudes de entrada únicamente el cromatógrafo usado $(p)$ y los MR utilizados ( $\mathrm{cal}$ ), la incertidumbre de las dos magnitudes se tomó como la declarada por los fabricantes, y se asume que los dos coeficientes de sensibilidad valen 1 .

La precisión de los resultados del PC del GN depende principalmente de las fuentes de incertidumbre como la composición del GN, el $Z$ y el $P C$ ideal asociados a su proceso de medición [63].Cruz de Oliveira [61] estimó la incertidumbre en el $Z$ y el $P C$ superior basándose en el método GUM. Para el $Z$ la estimación de incertidumbre fue determinada según las Ecuaciones 11 y 12.

$$
\begin{gathered}
u^{2}(Z)=\left(\frac{\partial Z}{\partial X_{j}} * u\left(X_{j}\right)\right)^{2}+\left(\frac{\partial Z}{\partial \beta_{i j}} * u\left(\beta_{i j}\right)\right)^{2} \\
u_{c}^{2}(Z)=\sum\left(-2 \sqrt{\beta_{i j}} \sum_{j=1}^{n} X_{j} \sqrt{\beta_{i j}}\right)^{2} * u^{2}\left(X_{j}\right)+\sum_{i=1}^{n}\left(-2 X_{i} \sum_{j=1}^{n} X_{j} \sqrt{\beta_{i j}}\right)^{2} * u^{2}\left(\beta_{i j}\right)
\end{gathered}
$$

Donde $X_{j}$ es la fracción molar del componente $j, n$ es el número total de componentes, y $\beta_{i j}$ es un parámetro que caracteriza la relación entre parejas de componentes con una incertidumbre correspondiente al $0,1 \%$ de $\sqrt{ } \beta_{i j}$. La incertidumbre del $P C_{s}$ volumétrico es estimada a partir de la Ecuación 13.

$$
u_{c}^{2}\left(P C_{s}\right)=\sum_{i}^{n}\left(\frac{1}{Z^{2}} u^{2}\left(P C_{\text {inf }}\right)_{i}\right)+\sum_{i}^{n}\left(\frac{\left(P C_{\text {inf }}^{2}\right)}{Z^{4}} u^{2}\left(Z_{i}\right)\right)
$$

Donde $P C_{\text {sup }}$ y $P C_{\text {inf }}$ corresponden al $P C_{s}$ y $P C_{i}$ respectivamente.

Skylogianni [64] comparó la predicción del PRHC obtenida por tres modelos termodinámicos diferentes con mediciones experimentales para dos gases sintéticos y uno real. Los modelos evaluados fueron la ecuación SRK, la ecuación PR con regla de mezclado universal (UMR-PRU), y la ecuación de estado PC-SAFT (Perturbed-Chain Statistical Associating Fluid Theory). Las predicciones de SRK y PC-SAFT son muy similares a presiones bajas hasta la temperatura cricondenterma y a temperaturas altas se comporta mejor SRK, mientras que el modelo UMR-PRU se comporta mejor al evaluar los gases sintéticos. Los autores realizaron un análisis de incertidumbre al aplicar UMR-PRU en la construcción de la envolvente de fases obteniendo una desviación máxima de $6,1 \mathrm{~K}$. Sin embargo, en los casos estudiados, las incertidumbres más bajas se presentan a bajas presiones y va aumentando a medida que aumenta la presión, haciendo el sistema más inestable. Hajipour et al. [65] plantearon que la estimación de incertidumbre del PRHC se podía cuantificar a partir de un algoritmo sencillo de propagación del error, relacionando los parámetros de interacción 
binaria y los parámetros del modelo termodinámico, combinado la simulación de MC con el método Latin Hypercube Sampling, y concuerdan con otros autores en que la incertidumbre de los parámetros de entrada afectan el cálculo del PRHC del GN. Adicionalmente, los autores estudiaron de forma particular la variabilidad presentada al inyectar n-Butano a una corriente de $\mathrm{GN}$, encontrando incertidumbres alrededor de $0,35 \mathrm{~K}$.

Martins et al. [66] realizaron un estudio de estimación de incertidumbre en la medición indirecta de la temperatura de rocío cuando se utiliza un generador de humedad de dos presiones como estándar de referencia. Los autores compararon tres enfoques: el método $\mathrm{MC}$, la inferencia bayesiana y el método GUM; con el primer enfoque obtuvieron incertidumbres del orden de $\pm 0,138 \mathrm{~K}$ y $\pm 0,196 \mathrm{~K}$ y con el enfoque bayesiano obtuvieron incertidumbres entre $\pm 0,124 \mathrm{~K}$ y $\pm 1,96 \mathrm{~K}$. Estos valores fueron comparados con los obtenidos por otros autores y finalmente aplicaron la metodología GUM para validar sus resultados, encontrando que a pesar de que los primeros dos enfoques son más rigurosos, la metodología GUM permite obtener resultados similares al método MC y a la literatura, usando las mismas condiciones y es mucho más sencilla de aplicar; sin embargo, proponen como trabajo posterior un estudio más profundo y detallado del método Bayesiano para aumentar la eficiencia de la estimación de incertidumbre.

Aparicio y Ayala [67] presentaron un análisis de identificación y cuantificación de las fuentes de incertidumbre involucradas en la medición del PRHC y el PC, a partir de la composición de un gas natural real proveniente de un yacimiento de Colombia. En dicho trabajo se comparan dos metodologías de estimación de incertidumbre: la metodología GUM y el método MC, para la medición del PC, obtenido de acuerdo a la norma GPA-2172 [67] y para el PRHC de acuerdo a dos ecuaciones de estado (PR y SRK) y dos reglas de mezclado (clásica y Huron-Vidal).

En cuanto al PC, la norma ISO 6976 [69] declara que en la práctica se reporta la estimación de la incertidumbre de un valor del PC en relación con algún punto de referencia (comúnmente el metano), en vez de reportar una incertidumbre absoluta de la medición, y que ésta se deriva de la determinación cromatográfica de la composición del GN.

\section{Discusión}

Debido a la dependencia de la medición indirecta de los parámetros de calidad de gas con respecto a la composición del GN, es de vital importancia que se realice una correcta determinación cromatográfica y estimación de incertidumbre de la misma, para obtener resultados analíticos con alta confiabilidad metrológica. En el caso del PC la precisión en su determinación es atribuida generalmente a tres contribuciones, las cuales estan dadas por las incertidumbres obtenidas en la determinación de cada uno de los componentes individuales de la mezcla de GN, el $Z$ y su composición. El valor del PRHC calculado puede variar de acuerdo a la ecuación de estado seleccionada y a la incertidumbre reportada en la literatura de las constantes críticas para cada compuesto. En realidad el PRHC es un conjunto de parejas de presión y temperatura que construyen la envolvente de fases, la cual muestra gráficamente el equilibrio entre la fase vapor y el condensado que corresponde a la fase líquida, por lo tanto, su medición debe involucrar el equilibrio de cada uno de los compuestos de la mezcla multicomponente en ambas fases. Pocos autores [62-64] han enfocado proyectos de investigación acerca de la determinación del PRHC y PC del gas natural y la estimación de su incertidumbre asociada; a pesar de ello, el PRHC ha recibido mayor atención debido a la necesidad de los transportadores de evitar daños en los equipos provocados por la condensación de los hidrocarburos en las líneas de GN. Por otro lado, a pesar de que la determinación del PC está basada en estándares internacionales, su estimación de incertidumbre es necesaria para complementar la medición y brindar confiabilidad metrológica a las mismas. No muchos trabajos reportan la incertidumbre del PC del GN, es por eso que se recomienda enfocar más investigaciones con respecto en ese tema con el fin de validar las mediciones realizadas de acuerdo al método.

Una correcta selección de la ecuación de estado, que represente adecuadamente el comportamiento de fases de la corriente particular de gas, conduce a una determinación de PRHC con un grado de incertidumbre bajo, y un mejor control sobre la posible presencia de condensados en el GN. Para este último fin, también es importante una adecuada selección de método de estimación de incertidumbre. 


\section{Conclusiones}

Este trabajo de revisión del estado del arte sobre la medición del PRHC y el PC del GN presenta las diferentes formas de medición de estos parámetros de calidad de gas, y diversos esfuerzos tendientes a estimar su incertidumbre de medición. Es evidente el grado de avance en cuanto a diferentes métodos de medición, tanto directos como indirectos, existiendo muchas técnicas bien establecidas y aceptadas para la cuantificación de PRHC y PC del gas natural. Sin embargo, es evidente la deficiencia en cuanto a estudios sobre la incertidumbre resultante de los resultados de medición de dichos parámetros de calidad, a pesar de la elevada importancia técnica y comercial tanto del de PRHC como del PC. La literatura relacionada con la estimación de la incertidumbre en la medición de los parámetros de calidad de gas es muy limitada.

En el caso del PC del gas natural, su medición indirecta en la industria se basa en normas internacionales, las cuales reportan un valor aproximado de incertidumbre determinado a partir de los datos experimentales considerados en la norma, que por consiguiente puede considerarse válido siempre y cuando la metodología descrita en el estándar se realice a cabalidad y se aplique a datos que queden incluidos en la región de valores de los datos experimentales en los que se basó la norma. Sin embargo, resulta conveniente que este tipo de mediciones lleven asociadas incertidumbres estimadas de acuerdo a los procedimientos y consideraciones que se tuvieron en cuenta en su determinación; de acuerdo a lo anterior, se han encontrado algunos estudios que aplican la metodología GUM para estimar la incertidumbre del PC cuando se mide de forma indirecta. En cuanto al PRHC, la mayoría de estudios se han orientado a la evaluación de la incertidumbre de mediciones directas en condiciones controladas, haciéndose evidente una falta de análisis de los valores reportados por mediciones indirectas (en cuanto a incertidumbre, además de tener en cuenta el sesgo) y de los valores obtenidos por determinación directa en condiciones más reales.

\section{Referencias bibliográficas}

[1] Liang FY, Ryvak M, Sayeed S, Zhao N. The role of natural gas as a primary fuel in the near future, including comparisons of acquisition, transmission and waste handling costs of as with competitive alternatives. Chem Cent J. 2012;6:S4.

[2] BP p.I.c. BP Statistical Review of World Energy June 2014. Londres. Inglaterra. 2014.

[3] Karpash O, Darvay I, Karpash M. New approach to natural gas quality determination. J Pet Sci Eng. 2010;71(3-4):133-7.

[4] ASTM International. D03 Committee. ASTM D1142: Test Method for Water Vapor Content of Gaseous Fuel by Measurement of Dew Point Temperature. 2012.

[5] Comisión de Regulación de Energía y Gas. Resolución CREG-071: Reglamento Único de Transporte de Gas Natural (RUT). Consejo Nacional de Operación de Gas Natural-CNO Gas. 1999.

[6] Comisión Reguladora de Energía (CRE). Norma Oficial Mexicana NOM-001SECRE-2003, Calidad del Gas Natural. Diario Oficial de la Federación. 2003.

[7] Australia Standards. Committee AG-010. AS 4564: Specification for general purpose natural gas. 2011.

[8] Bureau of Indian Standards. IS 15126:2002 Natural Gas -Standard Reference Conditions.

[9] Siemens AG. Process Analytics Throughout the Entire Natural Gas Pipeline Supply Chain. 2008.

[10] Ulbig P, Hoburg D. Determination of the calorific value of natural gas by different methods. Thermochim Acta. 2002;382(1-2):27-35.

[11] George DL, Barajas AM, Burkey RC. The need for accurate hydrocarbon dew point determination. Pipeline Gas J. 2005;232(9):32-4.

[12] Herring J. Hydrocarbon Dew Point Is A Critical Consideration For Pipeline Operations. Pipeline Gas J. 2010; 237(7):48.

[13]Meyer VR. Measurement uncertainty. J Chromatogr A. 2007;1158(1-2):15-24.

[14] Carotenuto A, Giovinco G, Viglietti B, Vanoli L. A new procedure for the determination of calibration curves for a gas chromatograph used in natural gas analysis. Chemom Intell Lab Syst. 2005;75(2):209-17.

[15] Haloua F, Ponsard JN, Lartigue G, Hay B, Villermaux $C$. Thermal behaviour modelling of a reference calorimeter for natural gas. Int $\mathrm{J}$ Therm Sci. 2012;55:40-7.

[16] Sunner S, Mansson M. Experimental chemical thermodynamics. 1: Combustion calorimetry. Oxford: Pergamon Press; 1979. 
[17]Domalski E S. From the history of combustion calorimetry. En: Experimental chemical thermodynamics. 1: Combustion calorimetry. Sunner S, Mansson M. Oxford: Pergamon Press; 1979. p. 401

[18]Popoff MM, Schirokich PK. Ein calorimeter zum verbrennen von chlor-und bromderivaten. Z.Phys. Chem (Leipzig). 1933;167:83.

[19] Haloua F, Ponsard J-N, Lartigue G, Hay $\mathrm{B}$, Villermaux $\mathrm{C}$, Foulon $\mathrm{E}$, et al. Thermal behaviour modelling of a reference calorimeter for natural gas. Int J Therm Sci. 2012;55:40-7.

[20] Barbato PS, Landi G, Russo G. Catalytic combustion of $\mathrm{CH}_{4}-\mathrm{H}_{2}-\mathrm{CO}$ mixtures at pressure up to 10bar. Fuel Process Technol. 2013;107:147-54.

[21]Pérez Fernando J, Sarge Stefan $M$. Development of an accurate, modern, adaptable, isothermal gas calorimeter. University of Valladolid and PTB. 2015

[22] Parameswaran T, Gogolek $P$, Hughes $P$. Estimation of combustion air requirement and heating value of fuel gas mixtures from flame spectra. Appl. Therm. Eng. 2016;105:353-61.

[23] Thompson ED. Chilled Mirror Device For Water \& Hydrocarbon Dew Point Determination. Chandler Engineering. AGMSC 2003.

[24] Tripathy S, Leong E-C, Rahardjo H. Total suction measurement of unsaturated soils with a device using the chilled-mirror dew-point technique. Géotechnique. 2003;53(2):173-82.

[25]Benton A. Determination of hydrocarbon dew point in natural gas. Am Sch Gas Meas Technol. 2006;1-8.

[26] Brown A, Milton M, Vargha G, Mounce R, Cowper C, Stokes A et al. NPL Report AS 3 Comparison of methods for the measurement of hydrocarbon dew point of natural gas. Inglaterra: National Physical Laboratory; 2007.

[27] American Petroleum Institute. Chapter 14-Natural Gas Fluids Measurements, section 1 Collecting and Handling of Natural Gas Samples for Custody Transfer. En: Manual of Petroleum Measurement Standards. Measurement Coordination Department. United States: API; 2006. p. 72.

[28] Joint Committee for Guides in Metrology (JCGM/WG 2). International vocabulary of metrology - Basic and general concepts and associated terms (VIM). BIPM. 2008.

[29]Barceló D. Advance techniques in gas chromatography -mass spectrometry (GCMS-MS and GC-TOF-MS) for environment chemistry. Elservier. 2013;61:360-5.

[30] Bahadori A. Natural gas processing: technology and engineering design. Amsterdam: Elsevier; 2014.

[31] Rojey A. Natural gas: production processing transport. Paris: Editions Technip; 1997. p. 429.

[32] American Gas Association. Report No.8: Compressibility Factors of Natural Gas and Other Related Hydrocarbon Gases. United States: Starling KE, Savidge JL. 1994.

[33] ASTM International. D03 Committee. ASTM 3588: Practice for Calculating Heat Value, Compressibility Factor, and Relative Density of Gaseous Fuels. 2011.

[34] Azizi N, Behbahani R, Isazadeh MA. An efficient correlation for calculating compressibility factor of natural gases. J Nat Gas Chem. 2010;19(6):642-5.

[35] Obuba J, Ikiesnkimama SS, Ubani CE, Ekeke IC. Natural Gas Compressibility Factor Correlation Evaluation for Niger Delta Gas Fields. IOSR-JEEE. 2013;6(4):10.

[36] Ahmed TH. Reservoir engineering handbook. 4th ed. Amsterdam: Gulf Professional Pub; 2010. p. 1454.

[37] Fayazi A, Arabloo M, Mohammadi AH. Efficient estimation of natural gas compressibility factor using a rigorous method. J Nat Gas Sci Eng. 2014;16:8-17.

[38] Kumar N. Compressibility factors for natural and sour reservoir gases by correlations and cubic equations of state (Tesis de maestría). United States. Texas Tech University. 2004.

[39]Zhou L, Zhou Y. Determination of compressibility factor and fugacity coefficient of hydrogen in studies of adsorptive storage. Int J Hydrog Energy. 2001;26(6):597-601.

[40] Heidaryan E, Moghadasi J, Rahimi M. New correlations to predict natural gas viscosity and compressibility factor. J Pet Sci Eng. 2010;73(1-2):67-72.

[41] Sanjari E, Lay EN. An accurate empirical correlation for predicting natural gas compressibility factors. J Nat Gas Chem. 2012;21(2):184-8.

[42] Kunz O, Wagner W. The GERG-2008 WideRange Equation of State for Natural Gases and Other Mixtures: An Expansion of GERG-2004. J Chem Eng Data. 2012;57(11):3032-91.

[43]Nasrifar K, Bolland O, Moshfeghian M. Predicting Natural Gas Dew Points from 15 Equations of State. Energy Fuels. 2005;19(2):561-72. 
[44]Nasrifar K, Bolland O. Prediction of thermodynamic properties of natural gas mixtures using 10 equations of state including a new cubic two-constant equation of state. J Pet Sci Eng. 2006;51(3-4):253-66.

[45]Elsharkawy AM. Predicting the dew point pressure for gas condensate reservoirs: empirical models and equations of state. Fluid Phase Equilibria. 2002;193(1-2):147-65.

[46] Galatro D, Marín-Cordero F. Considerations for the dew point calculation in rich natural gas. J Nat Gas Sci Eng. 2014;18:112-9.

[47] Jarrahian A, Heidaryan E. A new cubic equation of state for sweet and sour natural gases even when composition is unknown. Fuel. 2014;134:333-42.

[48] Cox MG, Harris PM. Measurement uncertainty and traceability. Meas Sci Technol. 2006;17(3):533-40.

[49] Taverniers I, De Loose M, Van Bockstaele E. Trends in quality in the analytical laboratory. I. Traceability and measurement uncertainty of analytical results. TrAC Trends Anal Chem. 2004;23(7):480-90.

[50]Wallace J. Ten methods for calculating the uncertainty of measurement. Sci Justice. 2010;50(4):182-6.

[51] Joint Committee for Guides in Metrology (JCGM/WG 1). Evaluation of measurement data - Guide to the expression of uncertainty in measurement. BIPM. 2008.

[52] Papadopoulos CE, Yeung $H$. Uncertainty estimation and Monte Carlo simulation method. Flow Meas Instrum. 2001;12(4):291-8.

[53] Joint Committee for Guides in Metrology (JCGM/WG 1). Evaluation of measurement data - Supplement 1 to the "Guide to the expression of uncertainty in measurement" -Propagation of distributions using a Monte Carlo method. BIPM. 2008.

[54]Enright PA, Fleischmann CM. Unvertainty of Heat Release Rate Calculation of the ISO5660-1 Cone Calorimeter Standard Test Method. Fire Technol. 1999;35(2):153-69.

[55]Zhao L, Dembsey NA. Measurement uncertainty analysis for calorimetry apparatuses. Fire Mater. 2008;32(1):1-26.

[56]Alexandrov YI. Estimation of the uncertainty for an isothermal precision gas calorimeters. Thermochim Acta. 2002;382(1):55-64.

[57] Haloua F, Foulon E, El-Harti E, Sarge SM,
Rauch J, Neagu $\mathrm{M}$, et al. Comparison of traceable methods for determining the calorific value of non-conventional fuel gases. Int. J. Therm. Sci. 2016;100:438-47.

[58] Yackow A. Laughton A, Gronemann U, Benito A, Lindgren T, Kukova E, et.al. GERGPROJECT 1.52 - Comparing and defining a relation between experimental and calculating techniques for hydrocarbon dew-point. International Gas Union Research Conference; 2008; París, Francia.

[59] Ramsey MH, Ellison SLR (Eds.). Eurachem/ EUROLAB/CITAC/Nordtest/AMC Guide: Measurement uncertainty arising from sampling: a guide to methods and approaches Eurachem. 2007. ISBN 9780948926266. Available from the Eurachem secretariat.

[60] Puglisi C. Kornblit F. Incertidumbre de medición en química analítica. Recta de calibración. Instituto Nacional de Tecnología Industrial; 2002.

[61]De Oliveira E. Simplified calibration methodology of chromatographs used in custody transfer measurements of natural gas. Metrol Meas Syst. 2012;19(2):405-16.

[62] Tsochatzidis NA, Karantanas E. Assessment of calorific value at a gas transmission network. J Nat Gas Sci Eng. 2012;9:45-50.

[63] Louli V, Pappa G, Boukouvalas C, Skouras $\mathrm{S}$, Solbraa $\mathrm{E}$, Christensen $\mathrm{KO}$, et al. Measurement and prediction of dew point curves of natural gas mixtures. Fluid Phase Equilibria. 2012;334:1-9.

[64] Skylogianni E. Measurements and Modelling of Hydrocarbon Dew Points for Natural Gases (Master thesis). Norway: Norwegian University of Science and Technology; 2012.

[65] Hajipour S, Satyro MA, Foley MW. Uncertainty Analysis Applied to Thermodynamic Models and Fuel Properties - Natural Gas Dew Points and Gasoline Reid Vapor Pressures. Energy Fuels. 2014;28(2):1569-78.

[66] Martins LL, Ribeiro AS, e Sousa JA, Forbes AB. Measurement Uncertainty of Dew-Point Temperature in a Two-Pressure Humidity Generator. Int J Thermophys. 2012;33(89):1568-82.

[67]Aparicio A, Ayala E. Identificación y Cuantificación de las Fuentes de Incertidumbre que Afectan el Proceso de Medición del Punto de Rocío de Hidrocarburo y el Poder Calorífico del Gas Natural. Met\&Flu. 10:06-15. 
[68]Gas Processor Association. GPA 2172: Calculation of Gross Heating Value, Relative Density, Compressibility and Theoretical Hydrocarbon Liquid Content for Natural Gas Mixtures for Custody Transfer. Washington,
D.C. United States of America. 2015.

[69] International Organization for Standardization. ISO6976: Calculation of calorific values, density, relative density and Wobbe index from composition. Ginebra, Suiza. ISO.1995. 